Research paper

\title{
Trait anxiety mediates the effect of stress exposure on post-traumatic stress disorder and depression risk in cardiac surgery patients
}

\author{
Lotte Kok $^{\mathrm{a}, *, 1}$, Milou S. Sep ${ }^{\mathrm{b}, 1}$, Dieuwke S. Veldhuijzen ${ }^{\mathrm{a}, \mathrm{c}}$, Sandra Cornelisse ${ }^{\mathrm{b}}$, \\ Arno P. Nierich ${ }^{\mathrm{d}}$, Joost van der Maaten ${ }^{\mathrm{e}}$, Peter M. Rosseel ${ }^{\mathrm{f}}$, Jan Hofland ${ }^{\mathrm{g}}$, \\ Jan M. Dieleman ${ }^{\mathrm{a}}$, Christiaan H. Vinkers ${ }^{\mathrm{h}}$, Marian Joëls ${ }^{\mathrm{b}}$, Diederik van Dijk ${ }^{\mathrm{a}}$, for the DECS \\ study group ${ }^{2}$, Manon $\mathrm{H}$. Hillegers ${ }^{\mathrm{h}}$
}

a Department of Anesthesiology and Intensive Care, Brain Center Rudolf Magnus, University Medical Center Utrecht, Utrecht, The Netherlands

${ }^{\mathrm{b}}$ Department of Translational Neuroscience, Brain Center Rudolf Magnus, University Medical Center Utrecht, Utrecht, The Netherlands

${ }^{\mathrm{c}}$ Institute of Psychology, Health, Medical and Neuropsychology Unit, Leiden University, Leiden, The Netherlands

${ }^{\mathrm{d}}$ Department of Anesthesiology, Isala Clinics, Zwolle, The Netherlands

e Department of Anesthesiology, University Medical Center Groningen, Groningen, The Netherlands

${ }^{\mathrm{f}}$ Department of Anesthesiology, Amphia Hospital, Breda, The Netherlands

${ }^{g}$ Department of Anesthesiology, Erasmus Medical Center, Rotterdam, The Netherlands

h Department of Psychiatry, Brain Center Rudolf Magnus, University Medical Center Utrecht, Utrecht, The Netherlands

\section{A R T I C L E I N F O}

\section{Article history:}

Received 20 March 2016

Received in revised form

18 June 2016

Accepted 10 July 2016

Available online 21 July 2016

Keywords:

Post-traumatic stress disorder

Depression

Intensive care unit

Cardiac surgery

Trait anxiety

Childhood trauma

\begin{abstract}
A B S T R A C T
Background: Post-traumatic stress disorder (PTSD) and depression are common after cardiac surgery. Lifetime stress exposure and personality traits may influence the development of these psychiatric conditions.

Methods: Self-reported rates of PTSD and depression and potential determinants (i.e., trait anxiety and stress exposure) were established 1.5 to 4 years after cardiac surgery. Data was available for 1125 out of 1244 (90.4\%) participants. Multivariable linear regressions were conducted to investigate mediating and/ or moderating effects of trait anxiety on the relationship between stress exposure, and PTSD and depression. Pre-planned subgroup analyses were performed for both sexes.

Results: PTSD and depression symptoms were present in $10.2 \%$ and $13.1 \%$ of the participants, respectively. Trait anxiety was a full mediator of the association between stress exposure and depression in both the total cohort and female and male subgroups. Moreover, trait anxiety partially mediated the relationship between stress exposure and PTSD in the full cohort and the male subgroup, whereas trait anxiety fully mediated this relationship in female patients. Trait anxiety did not play a moderating role in the total patient sample, nor after stratification on gender.

Limitations: The unequal distribution of male (78\%) and female patients (22\%) might limit the generalizability of our findings. Furthermore, risk factors were investigated retrospectively and with variable follow-up time.

Conclusions: In cardiac surgery patients, trait anxiety was found to be an important mediator of postoperative PTSD and depression. Prospective research is necessary to verify whether these factors are reliable screening measures of individuals' vulnerability for psychopathology development after cardiac surgery.
\end{abstract}

(c) 2016 Elsevier B.V. All rights reserved.

\footnotetext{
* Correspondence to: Department of Intensive Care Medicine, University Medical Center Utrecht, Heidelberglaan 100, 3584 CX Utrecht, The Netherlands. E-mail address: L.Kok-3@umcutrecht.nl (L. Kok).

1 These authors contributed equally to this work.

2 The members of the DECS study group are listed in Supplemental Digital Content 1
}

\section{Introduction}

Patients undergoing cardiac surgery are at high risk of developing post-traumatic stress disorder (PTSD) and depression. These problems occur in up to $15 \%$ and $20 \%$ of the patients, respectively (Griffiths et al., 2007; Tully, 2012), and may hamper full recovery (Granja et al., 2012; McKhann et al., 1997; Pirraglia et al., 1999).

Stress exposure during life may alter hypothalamic-pituitaryadrenal (HPA) axis responses when encountering novel stressful 
situations (Binder et al., 2008; Maripuu et al., 2014). Furthermore, stress exposure during childhood has long-term consequences on the ability to cope with stressors later in life (Horovitz et al., 2012). The influence of lifetime stress exposure on the development of psychopathology (i.e., PTSD and depression) in cardiac surgery patients is currently unknown.

The impact of lifetime stress on an individual depends partially on personality traits, such as anxiety (DiGangi et al., 2013; Kadak et al., 2013; Spielberger et al., 1983; van der Ploeg, 1980; van Reedt Dortland et al., 2012; Vinkers et al., 2014). High trait anxiety is common among cardiac patients and associated with core symptoms of PTSD (Jakšic et al., 2012; Jones et al., 2001; Kress et al., 2003). We hypothesized that the relation between lifetime stress exposure and psychopathology is mediated or moderated by the individual variation in trait anxiety. Identifying a mediating effect would imply trait anxiety to be a key factor in the causal pathway of lifetime stress exposure to psychopathology, and thus potentially amenable for interventions. A moderating role of trait anxiety would provide a more precise estimate of individual vulnerability to psychopathology after lifetime stress exposure and thereby contribute to our understanding of disease etiology (Kraemer et al., 2008).

The aim of this study was to investigate the mediating and/or moderating effect of trait anxiety on the relationship between childhood trauma and stressful life events on the one hand, and the post-operative development of PTSD and depression in cardiac surgery patients on the other hand.

\section{Materials and methods}

\subsection{Patients}

Patients of 18 years and older who were scheduled for cardiac surgery requiring cardiopulmonary bypass were eligible to participate in the Dexamethasone for Cardiac Surgery (DECS) study (Clinicaltrials.gov identifier: NCT00293592). DECS study participants were randomized to receive dexamethasone or placebo ( $1 \mathrm{mg} / \mathrm{kg}$ bodyweight) intravenously during surgery in a doubleblind way. Details of the study design have been published previously (Dieleman et al., 2012). For the present study, patients who participated in the DECS study a maximum of 3.5 years ago were recruited from the five cardiac surgery centers which yielded approximately $90 \%$ of the original DECS study sample: University Medical Center Utrecht, Isala Clinics, Amphia Hospital, University Medical Center Groningen, and Erasmus Medical Center. Participants $(n=2458)$ were assessed for eligibility between April 2006 and November 2011. Exclusion criteria were derived from the DECS study, and encompassed a life expectancy of 6 months or less, or an off-pump cardiac surgery procedure. There were no additional exclusion criteria for the present follow-up study. Time between cardiac surgery and the current assessment of lifetime and present psychopathology and postoperative psychopathology varied from 1.5 to 4 years.

\subsection{Data collection}

Participants received questionnaires by mail and a reminder by telephone, in order to increase the response rate. After obtaining written informed consent for the current study $(n=1244 ; 50.6 \%$ of the eligible participants of the DECS study sample), 1125 (90.4\%) patients returned questionnaires (Fig. 1). All questionnaires were scanned at once, and correspondence of digitized scores with the paper questionnaires was checked (in 5\% of randomly selected participants). The Medical Ethics Committee of the University Medical Center Utrecht approved this study.

\subsection{PTSD and depression}

Symptoms related to PTSD were measured by the Self-Report Inventory for PTSD (SRIP), which was previously well validated (van Zuiden et al., 2011, 2012) though not specifically after ICU treatment. The SRIP is a 22 item questionnaire which addresses DSM-IV-TR criteria of PTSD in the past four weeks (Hovens et al., 2000). It is rated on a 4-point (0-3) Likert scale, with higher scores indicating the presence of more symptoms. A literature-based cutoff score of 39 (sensitivity $=0.74$, specificity $=0.81$ ) was used to detect if above threshold symptoms of PTSD (for clarity, further referred to as PTSD) were present (van Zelst et al., 2003a; 2003b). Internal consistency is good with Cronbach's alpha scores ranging

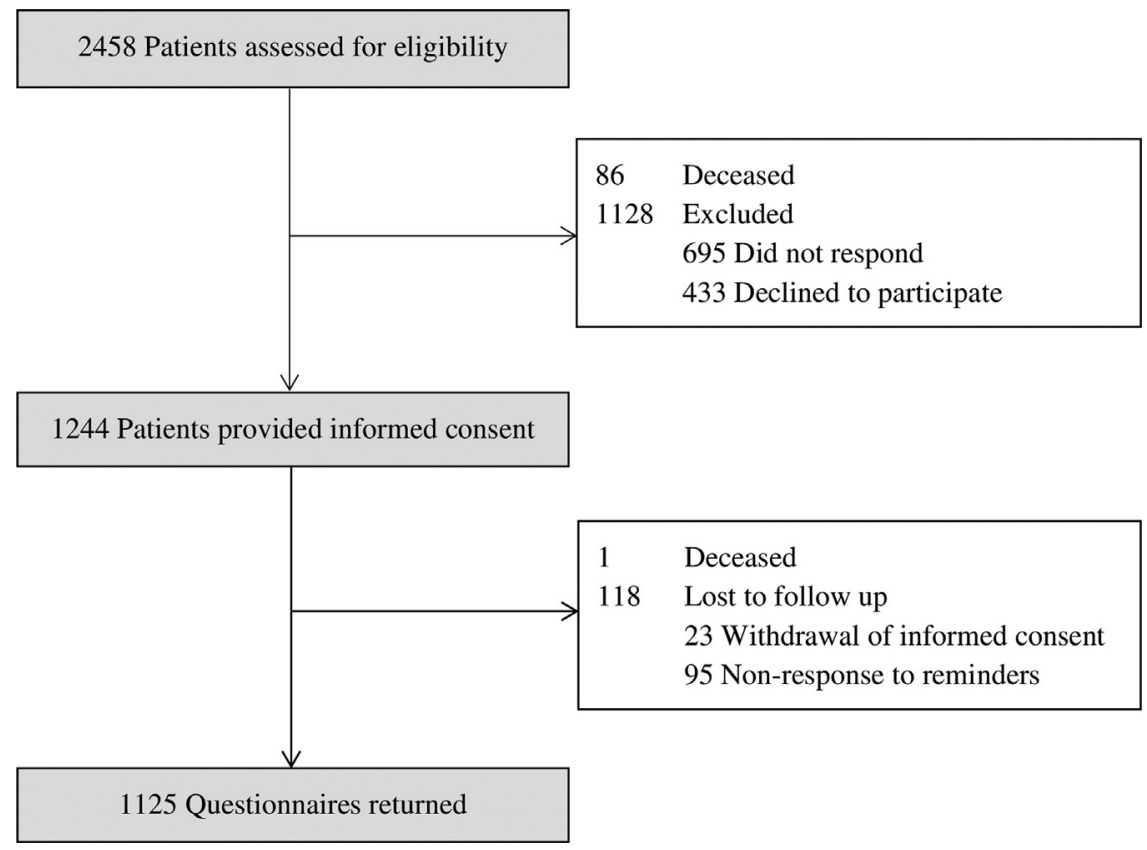

Fig. 1. Flow chart. 
between 0.90 and 0.94 ; average test-retest reliability is 0.69 (Hovens et al., 2000).

Depressive symptoms in the past week were measured conform DSM-IV-TR criteria by the 21-item Beck Depression Inventory - Revised (BDI-II) (Beck et al., 1996a). Each item scores on a scale from 0 to 3 and higher scores represent more severe symptoms. Depression was defined as the presence of above threshold symptoms (i.e., a cut-off score of 13.5 ; sensitivity $=0.81$, specificity $=0.92$ ) (Dozois et al., 1998). Cronbach's alpha and testretest reliability were 0.93 (Beck et al., 1996b).

\subsection{Stress exposure and trait anxiety}

The Childhood Trauma Questionnaire (CTQ) addresses five dimensions of trauma (i.e., physical abuse, emotional abuse, sexual abuse, physical neglect, and emotional neglect) during childhood and adolescence (Bernstein et al., 2003). It consists of 28 items, scored on a 5-point Likert scale. Dimension scores add up to yield a total score of 0 to 125 . Higher scores indicate a higher level of childhood trauma. Test-retest reliability is $0.79-0.86$, and median internal consistency reliability coefficients range from 0.66 to 0.92 (Thombs et al., 2009).

The exposure to life events before cardiac surgery was measured using the Life Stressor Checklist Revised (LSC-R). This questionnaire consists of 30 items and one point is assigned to every affirmative answer. This yields an overall life stressor score, ranging from 0 to 30 with higher scores representing exposure to more life events. Average test-retest reliability is 0.70 (Wolfe et al., 1996).

A comprehensive assessment of stressful experiences during life is more informative with respect to an individual's vulnerability for psychopathology than focusing on a single stress domain (Vinkers et al., 2014). Therefore, z-scores of CTQ and LSC-R outcomes were used to calculate a stress exposure score (Vinkers et al., 2014).

Trait anxiety was assessed using the State-Trait Anxiety Inventory Trait scale (STAI-T) (Kvaal et al., 2005). This 20-item questionnaire addresses individual differences in intensified state anxiety as reaction to potential threatening events (Barnes et al., 2002). It uses a 4-point Likert scale and yields a sum-score between 20 and 80, where higher scores indicate higher trait anxiety. Cronbach's alpha ranges from 0.73 to 0.97 and the average testretest reliability ranges between 0.73 and 0.86 (Barnes et al., 2002; Kvaal et al., 2005). High trait anxiety was defined in this study as a STAI-T score of 39 or higher (Kvaal et al., 2005).

\subsection{Other variables of interest}

The demographic characteristics age, sex, height, weight, level of education, and substance use were collected through the electronic hospital information system and follow-up questionnaires. These questionnaires also yielded retrospective information about coexisting psychiatric conditions (i.e., depression, anxiety disorder, and bipolar disorder) before surgery until 1.5 to 4 years post-operatively. Information about coexisting medical conditions were previously reported elsewhere (Kok et al., 2016). The DECS trial database contained data with regard to EuroSCORE (indicating the risk of perioperative mortality) (Nashef et al., 1999), length of ICU stay, first ICU admission, and prolonged mechanical ventilation (Dieleman et al., 2012).

\subsection{Statistical analysis}

Relevant socio-demographic and clinical characteristics were analyzed by using descriptive statistics. Missing values occurred in 274 cases (24.4\%), of which 58 missing outcome variables (5.2\%; 12 for PTSD and 46 for depression). With regard to baseline variables, missing values ranged from $0.0 \%$ (age, sex, ICU length of stay, depression, anxiety, and bipolar disorder) to $1.9 \%$ (level of education). Since biased inferences could result from conducting complete cases analysis only, multiple imputation was conducted using both independent and dependent variables. Analyses were conducted in ten imputed datasets and results were pooled according to Rubin's rule (Groenwold et al., 2012; Donders et al., 2006; Little, 1992; Rubin 1987).

We applied multivariable linear regression to quantify the possible association between stress exposure, and postoperative PTSD and depression. All potential important factors were assessed with regard to relevance, possible clinical implications, and accuracy of measurement. Power analysis conceded a maximum of six factors (i.e. age, sex, randomization variable, pre-existing psychopathology, stress exposure, and trait anxiety) that could be added to the regression model. To assess the role of trait anxiety, moderating and mediating analyses were conducted (Baron and Kenny, 1986; Koeske and Koeske, 1993). The independent and potential mediating/moderating determinants were centered by subtracting the sample means from each participant's values, to obtain z-scores (Aiken and West, 1991). Spearman rank correlation was used to assess univariate associations between all determinants. Significant associations are a precondition for a test of mediation (Holmbeck, 1997). To investigate the potential mediating effect of trait anxiety, a priori defined hierarchical regression was conducted on continuous SRIP and BDI-II scores. Age, gender, pre-existing psychopathology, and dexamethasone intervention (i.e., whether a patient received dexamethasone or placebo) were potential confounders and entered in step 1, stress exposure was regressed on step 2, and trait anxiety on step 3. Reduction of the $\beta$-value for the stress exposure variable from significance to nonsignificance in step 3 demonstrates full mediation (Peñacoba-Puente et al., 2008). Partial mediation by trait anxiety is present when the $\beta$ value is reduced, but not to non-significance (Peñacoba-Puente et al., 2008). To test the possible moderating effect of trait anxiety, the aforementioned potential confounders were entered in the first block, and stress exposure and trait anxiety in the second block. In the third block an interaction term -created by multiplying the centered stress exposure and moderator variables- was regressed. Regression lines were plotted when the main effect of this interaction term was significant. A moderating effect is present when the intercepts and slopes show change in the expected direction for regression equations with low and high values of trait anxiety.

Pre-planned subgroup analyses were carried out for both sexes. Complete case analysis was performed as a sensitivity analysis. A significance level of $\alpha=0.05$ was applied and IBM SPSS version 20 (SPSS Inc., Chicago, Ill) was used.

\section{Results}

\subsection{Sample characteristics}

Preoperative, surgical, and ICU-related baseline characteristics are listed in Table 1. According to the established cut-off scores, symptoms of PTSD and depression were present in respectively $10.2 \%$ and $13.1 \%$ of the study sample. High trait anxiety was present in 183 (16.3\%) patients, of which 120 (65.6\%) patients showed psychopathology.

\subsection{Mediating effect of trait anxiety on psychopathology}

Preconditions for a test of mediation were met. Medians, interquartile ranges, and Spearman rank correlation coefficients are listed in Table 2.

After addition of trait anxiety to the model, partial mediation on the relation between stress exposure and PTSD was found ( $\beta$ 
Table 1

Demographic and clinical characteristics ${ }^{\mathrm{a}}$.

\begin{tabular}{ll}
\hline Age, median (IQR), y & $69.6(63.0-76.1)$ \\
Male sex & $878(78.0)$ \\
Height, median (IQR), cm & $175(170-181)$ \\
Weight, median (IQR), kg & $82(73-92)$ \\
Level of education & \\
$\quad$ No education/elementary school & $224(19.9)$ \\
Lower vocational education & $395(35.1)$ \\
Higher vocational education & $433(38.5)$ \\
University & $73(6.5)$ \\
Intoxications & \\
Smoking & $105(9.3)$ \\
Alcohol & $876(77.9)$ \\
Substance use & $5(0.4)$ \\
Lifetime and present psychopathology & \\
Depression & \\
Anxiety disorder & $22(2.0)^{\mathrm{c}} / 26(2.3)^{\mathrm{d}}$ \\
$\quad$ Bipolar disorder & $18(1.6)^{\mathrm{c}} / 29(2.6)^{\mathrm{d}}$ \\
EuroSCORE ${ }^{\mathrm{e}}$ median (IQR) & $6(0.5)^{\mathrm{c}} / 1(0.1)^{\mathrm{d}}$ \\
ICU LOS, median (IQR), h & $4(3-6)$ \\
First ICU admission & $21(19-24)$ \\
Prolonged mechanical ventilation $(>48 \mathrm{~h})$ & $911(81.0)$ \\
\hline
\end{tabular}

Abbreviations: EuroSCORE, European System for Cardiac Operative Risk Evaluation; COPD, chronic obstructive pulmonary disease; ICU, intensive care unit; IQR, interquartile range; LOS, length of stay.

${ }^{*} p<.05$.

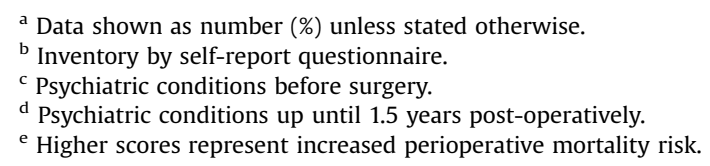

reduction from 0.325 to 0.068 and $p<.001$ to $p=0.003$; Fig. 2a; Table 3$)$. Trait anxiety fully mediated the effect between stress exposure and depression ( $\beta$ reduction from 0.282 to 0.015 and $p<0.001$ to $p=0.507$; Fig. 2a; Table 4). Subgroup analysis indicated trait anxiety to be a partial mediator of the effect between stress exposure and PTSD in male patients $(n=878$; Supplemental Table 1), whereas full mediation was found in female patients $(\mathrm{n}=247$; Supplemental Table 2). With regard to the relationship between stress exposure and depression, full mediation by trait anxiety was observed in both male and female patient subgroups (Supplemental Tables 3 and 4, respectively). Complete case analyses yielded comparable results.

\subsection{Moderating effect of trait anxiety on psychopathology}

Assessment of the moderating effect of trait anxiety on the relationship between stress exposure and PTSD showed a significant main effect for the interaction term [trait anxiety $x$ stress exposure] ( $\beta=0.051 ; p=0.038$ ). The intercepts and slopes for the high and low trait anxiety groups did not differ and did not change in the predicted direction (Fig. 3). For depression, no moderating effect of trait anxiety was found on the association between stress exposure and depression; the effect of the interaction term was non-significant $(\beta=0.008 ; p=0.754)$. Furthermore, stratified analysis for sex showed no moderating effect of trait anxiety on the relation between stress exposure, and PTSD and depression in male patients $(\beta=0.047 ; p=0.098$ and $\beta=0.011 ; p=0.694$, respectively), nor in female patients $(\beta=0.092 ; p=0.061$ and $\beta=0.015 ; p=0.747$, respectively). Complete case analyses yielded comparable results.

\section{Discussion}

This study is one of the largest observational studies evaluating PTSD and depression after cardiac surgery. We aimed to investigate the effect of trait anxiety on the relationship between childhood trauma and stressful life events, and the development of PTSD and depression.

Trait anxiety and stress exposure during life emerged as important risk factors for the development of psychopathology. More specifically, trait anxiety was found to mediate the impact of stress exposure during life on the risk to develop PTSD and depression. These findings might have clinical implications for the early identification of individuals at risk after cardiac surgery.

\subsection{Stress exposure during life and vulnerability to psychopathology}

During childhood, the neuronal system is particularly susceptible to environmental inputs, which can lead to functional and epigenetic modifications (Agorastos et al., 2014). Moreover, it affects fear response circuits and leads to long-lasting alteration in psychophysiological reactivity (Horovitz et al., 2012). Hence, the ability to cope with stressors later in life is hampered and associations with depression and PTSD are often found (Horovitz et al., 2012; Spindler, 2005).

In cardiac surgery patients, psychiatric morbidity is common and an increase of both symptom frequency and severity with regard to PTSD and depression is often reported after discharge (Musselman et al., 1998; Spindler, 2005). Vice-versa, the pathophysiologic progression of cardiovascular disease is influenced by the presence of depression: depressed patients have an increased risk for future cardiac events and mortality (Gold, 2015; Musselman et al., 1998). Furthermore, the presence of depressive symptoms negatively influences compliance with medical therapy and rehabilitation (Gold, 2015). Therefore, early recognition of psychopathology and effective treatment is crucial for recovery.

Table 2

Medians, interquartile range (IQR), and Spearman rank correlation coefficients for each of the assessed variables and outcome measures.

\begin{tabular}{|c|c|c|c|c|c|c|}
\hline & Median (IQR) & 2. LSC-R & 3. Stress exposure & 4. STAI-T & 5. SRIP & 6. BDI-II \\
\hline 1. CTQ & $33.0(30.0-38.0)^{a}$ & $.077^{*}$ & $.948^{\text {*** }}$ & $.335^{* * *}$ & $.269 * *$ & $.228^{* * *}$ \\
\hline 2. LSC-R & $3.0(2.0-4.0)^{\mathrm{a}}$ & - & $.365^{* * *}$ & $.147^{* * *}$ & $.196^{* * * k}$ & $.167^{* * *}$ \\
\hline 3. Stress exposure & $-2.5(-5.5-2.5)^{\mathrm{b}}$ & & - & $.340 * *$ & $.290^{* * *}$ & $.248^{* * *}$ \\
\hline 4. STAI-T & $28.0(24.0-36.0)$ & & & - & $.705^{\text {*k* }}$ & $.665^{* k \cdots}$ \\
\hline 5. SRIP & $26.0(23.0-31.0)$ & & & & - & $.751^{* * *}$ \\
\hline 6. BDI-II & $5.0(2.0-9.0)$ & & & & & - \\
\hline
\end{tabular}

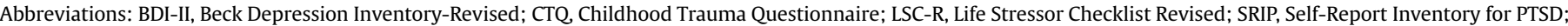
STAI-T, State-Trait Anxiety Inventory Trait scale.

$$
\begin{aligned}
& \begin{array}{l}
* \\
* *
\end{array}<05 \\
& \text { * } p<.01 \\
& \text { a scores centered by subtracting the sample means from each participant's values } \\
& { }^{\mathrm{b}} \text { Centered variable composed by adding up centered CTQ and LSC-R scores. }
\end{aligned}
$$


a

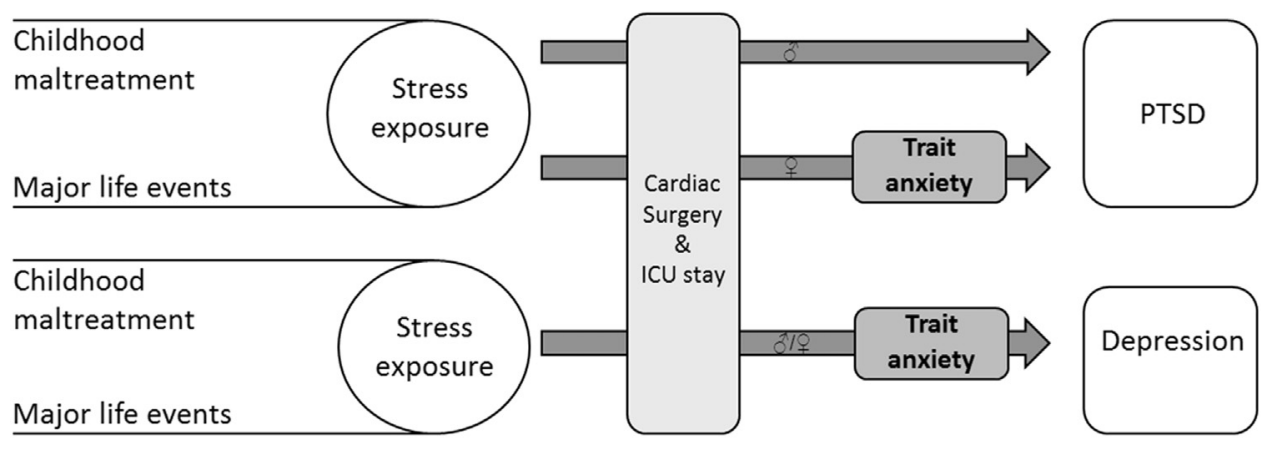

b

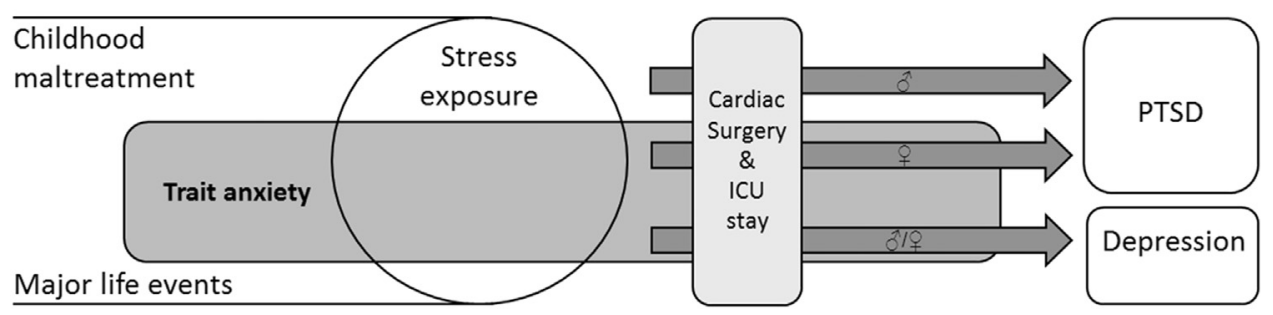

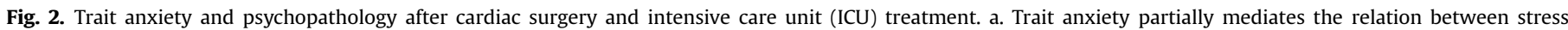

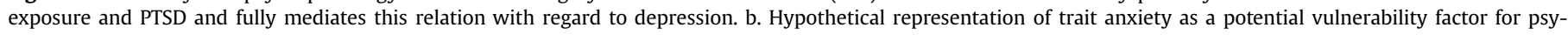
chopathology after exposure to cardiac surgery and intensive care unit (ICU) treatment.

\subsection{Trait anxiety and coping with stress}

In line with previous studies (Jakšic et al., 2012; Jones et al., 2001; Peñacoba-Puente et al., 2008), we found trait anxiety to be the strongest determinant for PTSD and depression. People with high trait anxiety have a tendency to perceive stressful situations as dangerous or threatening (Spielberger et al., 1983). It has been shown that these individuals have a more sensitive autonomic system and their arousal levels take longer to return to baseline (Jakšic et al., 2012; van der Ploeg, 1980). This indicates that trait anxiety affects the way in which patients are able to process and cope with stressful events, ultimately affecting their mental health (Jakšic et al., 2012; van der Ploeg, 1980). The hypothetical role of high trait anxiety as vulnerability factor for developing psychopathology is depicted in Fig. 2b. We further explored the role of these affective-cognitive responses (Hong and Paunonen, 2011) and found trait anxiety to have a partial mediating effect on PTSD, whereas full mediation was seen with regard to depression. This difference might be due to the fact that an exposure to a stressful event is inherent to PTSD, which is not the case for depression (American Pyschiatric Association, 2000).

Post-hoc subgroup analyses did not yield different results between male and female patients, except for the mediating effect of trait anxiety with regard to PTSD. Since our cohort consisted predominantly of male patients, these observed effects in the total sample could be driven by the male subgroup. Since gender

Table 3

Associations between stress exposure, trait anxiety and PTSD.

\begin{tabular}{|c|c|c|c|c|c|c|c|}
\hline & & $\beta$ & $t$ & $F(\mathrm{df})$ & $R^{2}$ & Adjusted $R^{2}$ & $p$-value \\
\hline \multirow[t]{5}{*}{ Step 1: } & Age & .009 & .288 & & & & .774 \\
\hline & Gender & -.061 & -1.892 & & & & .059 \\
\hline & Randomization variable & -.024 & -.758 & & & & .449 \\
\hline & Pre-existing psychopathology & -.034 & -1.081 & & & & .280 \\
\hline & & & & $1.438(4)$ & .006 & .002 & \\
\hline \multirow[t]{6}{*}{ Step 2: } & Age & .011 & 0.376 & & & & .707 \\
\hline & Gender & -.054 & -1.790 & & & & .074 \\
\hline & Randomization variable & -.031 & -1.026 & & & & .305 \\
\hline & Pre-existing psychopathology & -.022 & -.713 & & & & .476 \\
\hline & Stress exposure & .325 & 10.829 & & & & .000 \\
\hline & & & & $24.739(5)$ & .111 & .107 & \\
\hline \multirow[t]{7}{*}{ Step 3: } & Age & .023 & 1.080 & & & & .281 \\
\hline & Gender & -.026 & -1.191 & & & & .234 \\
\hline & Randomization variable & -.076 & -3.512 & & & & .000 \\
\hline & Pre-existing psychopathology & -.021 & -.994 & & & & .320 \\
\hline & Stress exposure ${ }^{a}$ & .068 & 2.962 & & & & .003 \\
\hline & Trait anxiety & .711 & 30.791 & & & & .000 \\
\hline & & & & 198.391(6) & .547 & .544 & \\
\hline
\end{tabular}

Note: Standardized regression coefficients (betas) are derived from the step in which they are added to the equation. PTSD, post-traumatic stress disorder.

a Sum of centered scores on childhood trauma and stressful life events. 
Table 4

Associations between stress exposure, trait anxiety and depression.

\begin{tabular}{|c|c|c|c|c|c|c|c|}
\hline & & $\beta$ & $t$ & $F(\mathrm{df})$ & $R^{2}$ & Adjusted $R^{2}$ & $p$-value \\
\hline \multirow[t]{5}{*}{ Step 1: } & Age & .043 & 1.337 & & & & .181 \\
\hline & Gender & -.078 & -2.432 & & & & .015 \\
\hline & Randomization variable & .015 & .464 & & & & .643 \\
\hline & Pre-existing psychopathology & -.004 & -.131 & & & & .895 \\
\hline & & & & $1.804(4)$ & .007 & .003 & \\
\hline \multirow[t]{6}{*}{ Step 2: } & Age & .045 & 1.455 & & & & .146 \\
\hline & Gender & -.072 & -2.355 & & & & .019 \\
\hline & Randomization variable & .009 & .291 & & & & .771 \\
\hline & Pre-existing psychopathology & .007 & .230 & & & & .819 \\
\hline & Stress exposure $\mathrm{a}^{\mathrm{a}}$ & .282 & 9.257 & & & & .000 \\
\hline & & & & $18.704(5)$ & .086 & .082 & \\
\hline \multirow[t]{7}{*}{ Step 3: } & Age & .057 & 2.664 & & & & .008 \\
\hline & Gender & -.043 & -1.996 & & & & .046 \\
\hline & Randomization variable & -.038 & -1.763 & & & & .078 \\
\hline & Pre-existing psychopathology & .007 & .332 & & & & .740 \\
\hline & Stress exposure & .015 & .664 & & & & .507 \\
\hline & Trait anxiety & .738 & 32.263 & & & & .000 \\
\hline & & & & $205.472(6)$ & .555 & .553 & \\
\hline
\end{tabular}

Note: Standardized regression coefficients (betas) are derived from the step in which they are added to the equation.

a Sum of centered scores on childhood trauma and stressful life events.

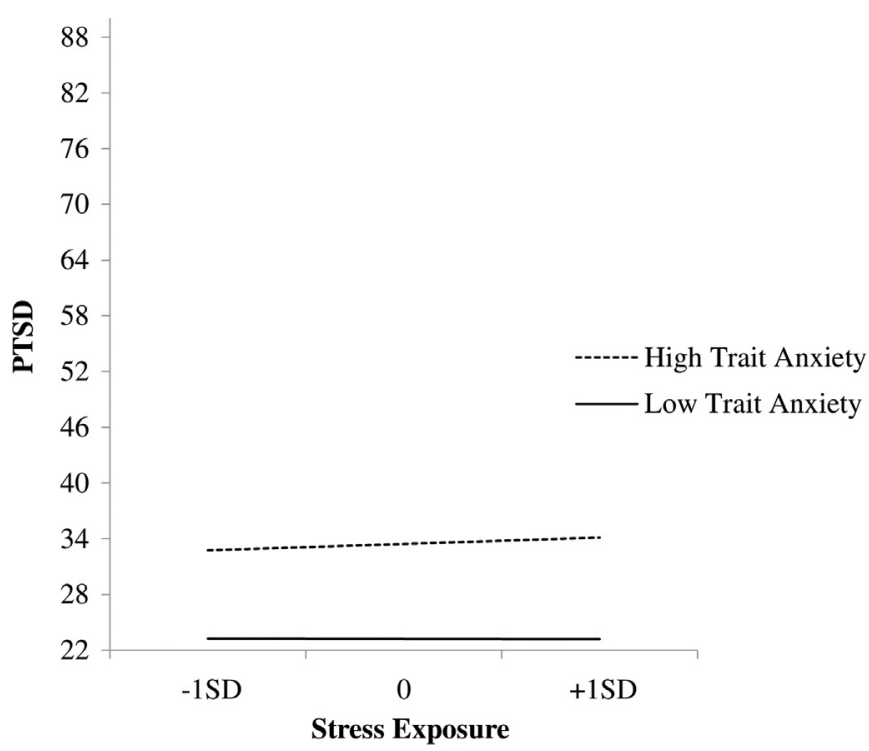

Fig. 3. Moderating effects of trait anxiety between stress exposure and symptoms of post-traumatic stress disorder (PTSD) as measured by the Self-Report Inventory for PTSD (SRIP). Regression lines are plotted for high ( +1 SD above the mean) and low $(-1$ SD below the mean) values of stress exposure.

differences are not uncommon in anxiety and mood disorders (Bouchoucha et al., 2013; Solomon and Herman, 2009; Viswanath et al., 2011), future studies with a prospective design and a more equal female/male distribution are necessary to elaborate on our preliminary findings.

\subsection{Strengths \& limitations}

This study has several important strengths. We studied potential risk factors for the development of psychopathology in a large cohort of homogeneous cardiac surgery patients. The short validated questionnaires hold potential for use as screening instruments in a clinical setting. The long-term follow up period of 1.5 until 4 years after surgery adds important information to the existing literature, which predominantly describes psychopathology within 1 year after discharge. Furthermore, it was previously shown that there were no differences in the occurrence of PTSD and depressive symptoms throughout this variable follow-up time period (Kok et al., 2016).

However, the study data should be interpreted with the following limitations in mind. First, the study population included more male (78\%) than female patients (22\%) which is typical for a cardiac surgery cohort (Bening et al., 2013) but might limit the generalizability of our findings. Second, the presence of PTSD and depressive symptoms is based on validated questionnaires by mail instead of a diagnostic interview (Hovens et al., 2000; van Zelst et al., 2003b; Beck et al., 1996a, 1996b). Therefore, our outcome measurements merely give an indication of the presence of PTSD and depression symptoms. Moreover, this cohort only allowed us to investigate risk factors retrospectively and with variable followup time. Although no significant differences were found with regard to follow-up time, this makes our results more difficult to interpret. In theory, trait anxiety and childhood trauma scores should be similar whether tested prior to or after surgery since these are considered to remain relatively stable over time (van der Ploeg, 1980). However, in previous literature an association between childhood trauma scores and psychopathology is more frequently found in retrospective studies in comparison to prospective studies (Scott et al., 2012). Therefore, a certain degree of recall bias cannot be ruled out. Lastly, a selection bias based on higher rates of cognitive impairment or mental health problems in the non-responders cannot not be ruled out, since information about pre- and postoperative cognitive functioning or psychopathology (i.e. up to 1.5 years postoperatively) was not assessed.

\section{Conclusion and clinical implications}

We found trait anxiety to be a potent mediator of the relation between stress exposure, and the development of PTSD and depression after cardiac surgery. Screening on trait anxiety might provide quickly available and valuable information and identify those most in need for psychological monitoring after discharge.

\section{Conflict of interest}

The authors declare no conflict of interest. 


\section{Contributors}

Diederik van Dijk, Marian Joëls, Manon Hillegers, Christiaan Vinkers, and Sandra Cornelisse designed the study and wrote the protocol. Arno Nierich, Joost van der Maaten, Peter Rosseel, Jan Hofland, and Jan Dieleman contributed to the management of inclusion of patients and collecting of data. Lotte Kok and Milou Sep wrote the first draft, managed the literature searches and analyses, supervised by Dieuwke Veldhuijzen and Manon Hillegers.

\section{Role of the funding source}

Funding for this follow up study was provided by a personal grant from the Netherlands Foundation for Mental Health (Fonds Psychische gezondheid, project 201126672, Postoperative psychopathology after cardiac surgery: Effects of dexamethasone and relation with corticosteroid receptor single nucleotide polymorphisms) to MHJ Hillegers. The DECS trial itself was supported by grants 80-82310-98-08607 from the Netherlands Organization for Health Research and Development (ZonMw) and 2007B125 from the Dutch Heart Foundation.

\section{Acknowledgments}

The authors thank Cor Kalkman, Sandra Numan, Linda Peelen and Wietze Pasma for their valuable input and help with organizational, administrative, statistical, and technical challenges.

\section{Appendix A. Supporting information}

Supplementary data associated with this article can be found in the online version at http://dx.doi.org/10.1016/j.jad.2016.07.020.

\section{References}

Agorastos, A., Pittman, J.O.E., Angkaw, A.C., Nievergelt, C.M., Hansen, C.J., Aversa, L H., Parisi, S.A., Barkauskas, D.A., Marine Resiliency Study Team, Baker, D.G., 2014. The cumulative effect of different childhood trauma types on self-reported symptoms of adult male depression and PTSD, substance abuse and health-related quality of life in a large active-duty military cohort. J. Psychiatr. Res. 58, 46-54.

Aiken, L.S., West, S.G., 1991. Multiple Regression: Testing and Interpreting Interactions. SAGE publications, Inc, Newbury Park, CA.

American Psychiatric Association, 2000. DSM-IV-TR. Diagnostic and Statistical Manual of Mental Disorders 4th edition TR.

Barnes, L.L.B., Harp, D., Lung, W.S., 2002. Reliability generalization of scores on the spielberger state-trait anxiety inventory. Educ. Psychol. Meas. 62, 603-618.

Baron, R.M., Kenny, D.A., 1986. The moderator-mediator variable distinction in social psychological research: conceptual, strategic, and statistical considerations. J. Personal. Soc. Psychol. 51, 1173-1182.

Beck, A.T., Steer, R.A., Ball, R., Ranieri, W.F., 1996. Comparison of Beck Depression Inventories-IA and-II in psychiatric outpatients. J. Personal. Assess. 67, 588-597.

Beck, A., Steer, R., Brown, G., 1996b. BDI-II, Beck depression inventory: manual for Beck Depression Inventory-II.

Bening, C., Weiler, H., Vahl, C.-F., 2013. Effects of gender, ejection fraction and weight on cardiac force development in patients undergoing cardiac surgery an experimental examination. J. Cardiothorac. Surg. 8, 214.

Bernstein, D.P., Stein, J.A., Newcomb, M.D., Walker, E., Pogge, D., Ahluvalia, T., Stokes, J., Handelsman, L., Medrano, M., Desmond, D., Zule, W., 2003. Development and validation of a brief screening version of the childhood trauma questionnaire. Child Abus. Negl. 27, 169-190.

Binder, B.C., Bradley, R.G., Liu, W., Epstein, M.P., Deveau, T.C., Mercer, K.B., Tang, Y., Gillespie, C.F., Heim, C.M., Nemeroff, C.B., Schwartz, A.C., Cubells, J.F., Ressler, K. J., 2008. Association of FKBP5 polymorphisms and childhood abuse with risk of posttraumatic stress disorder symptoms in adults. JAMA 299, 1291-1305.

Bouchoucha, M., Hejnar, M., Devroede, G., Babba, T., Bon, C., Benamouzig, R., 2013. Anxiety and depression as markers of multiplicity of sites of functional gastrointestinal disorders: a gender issue? Clin. Res Hepatol. Gastroenterol. 37, 422-430.

Dieleman, J.M., Nierich, A.P., Rosseel, P.M., van der Maaten, J.M., Hofland, J., Diephuis, J.C., Schepp, R.M., Boer, C., Moons, K.G., Herwerden, L.A., Tijssen, J.G.,
Numan, S.C., Kalkman, C.J., van Dijk, D., 2012. Intraoperative high-dose dexamethasone for cardiac surgery: a randomized controlled trial. JAMA 308, 1761-1767.

DiGangi, J.A., Gomez, D., Mendoza, L., Jason, L.A., Keys, C.B., Koenen, K.C., 2013. Pretrauma risk factors for posttraumatic stress disorder: a systematic review of the literature. Clin. Psychol. Rev. 33, 728-744.

Donders, A.R.T., van der Heijden, G.J.M.G., Stijnen, T., Moons, K.G.M., 2006. Review: a gentle introduction to imputation of missing values. J. Clin. Epidemiol. 59, 1087-1091.

Dortland, Van Reedt, Giltay, A.K.B., van Veen, E.J., Zitman, T., Penninx, F.G., J.H, B.W. 2012. Personality traits and childhood trauma as correlates of metabolic risk factors: The Netherlands Study of Depression and Anxiety (NESDA). Prog. Neuro-Psychopharmacol. Biol. Psychiatry 36, 85-91.

Dozois, D.J.A., Dobson, K.S., Ahnberg, J.L., 1998. A psychometric evaluation of the Beck Depression Inventory-II. Psychol. Assess. 10, 83-89.

Gold, P.W., 2015. The organization of the stress system and its dysregulation in depressive illness. Mol. Psychiatry 20, 32-47.

Granja, C., Amaro, A., Dias, C., Costa-Pereira, A., 2012. Outcome of ICU survivors: a comprehensive review. the role of patient-reported outcome studies. Acta Anaesthesiol. Scand. 56, 1092-1103.

Griffiths, J., Fortune, G., Barber, V., Young, J.D., 2007. The prevalence of post traumatic stress disorder in survivors of ICU treatment: a systematic review. Intensive Care Med. 33, 1506-1518.

Groenwold, R.H.H., Donders, A.R.T., Roes, K.C.B., Harell Jr., F.E., Moons, K.G.M., 2012 Dealing with missing outcome data in randomized trials and observational studies. Am. J. Epidemiol. 175, 210-217.

Holmbeck, G.N., 1997. Toward terminological, conceptual, and statistical clarity in the study of mediators and moderators: examples from the child-clinical and pediatric psychology literatures. J. Consult Clin. Psychol. 65, 599-610.

Hong, R., Paunonen, S., 2011. Personality vulnerabilities to psychopathology: relations between trait structure and affective-cognitive processes. J. Personal. 79, 527-562.

Horovitz, O., Tsoory, M.M., Hall, J., Jacobson-Pick, S., Richter-Levin, G., 2012. Postweaning to pre-pubertal (juvenile) stress: a model of induced predisposition to stress-related disorders. Neuroendocrinology 95, 56-64.

Hovens, J.E., van der Ploeg, H.M., Bramsen, I., Reuling, I.E., 2000. Test-retest reliability of the self-rating inventory for posttraumatic stress disorder. Psychol. Rep. 87, 735-737.

Jakšic, N., Brajković, L., Ivezić, E., Topić, R., Jakovljević, M., 2012. The role of personality traits in posttraumatic stress disorder (PTSD). Psychiatr. Danub. 24, 256-266.

Jones, C., Griffiths, R.D., Humphris, G., Skirrow, P.M., 2001. Memory, delusions, and the development of acute posttraumatic stress disorder-related symptoms after intensive care. Crit. Care Med. 29, 573-580.

Kadak, M.T., Nasiroglu, S., Boysan, M., Aydin, A., 2013. Risk factors predicting posttraumatic stress reactions in adolescents after 2011 Van earthquake. Compr. Psychiatry 54, 982-990.

Koeske, G.F., Koeske, R., 1993. A preliminary test of a stress-outcome model for reconceptualizing the burnout phenomenon. J. Soc. Serv. Res. 17, 107-128.

Kok, L., Hillegers, M.H., Veldhuijzen, D.S., Cornelisse, S., Nierich, A.P., van der Maaten, J.M., Rosseel, P.M., Hofland, J., Sep, M.S., Dieleman, J.M., Vinkers, C.H. Peelen, L.M., Joëls, M., van Dijk, D., 2016. The effect of dexamethasone on symptoms of posttraumatic stress disorder and depression after cardiac surgery and intensive care admission: longitudinal follow-up of a randomized controlled trial. Crit. Care Med. 44, 512-520.

Kraemer, H.C., Kiernan, M., Essex, M., 2008. How and why criteria defining moderators and mediators differ between the Baron \& Kenny and MacArthur approaches. Health Psychol. 27, S101-S108.

Kress, J.P., Gehlbach, B., Lacy, M., Pliskin, N., Pohlman, A.S., Hall, J.B., 2003. The longterm psychological effects of daily sedative interruption on critically ill patients. Am. J. Respir. Crit. Care Med. 168, 1457-1461.

Kvaal, K., Ulstein, I., Nordhus, I.H., Engedal, K., 2005. The Spielberger State-Trait Anxiety Inventory (STAI): the state scale in detecting mental disorders in geriatric patients. Int. J. Geriatr. Psychiatry 7, 629-634.

Little, R.J.A., 1992. Regression with missing x's: a review. J. Am. Stat. Assoc. 87, 1227-1237.

Maripuu, M., Wikgren, M., Karling, P., Adolfsson, R., Norrback, K.F., 2014. Relative hypo- and hypercortisolism are both associated with depression and lower quality of life in bipolar disorder: a cross-sectional study. PLoS One 9, 1-12.

McKhann, G.M., Borowicz, L.M., Goldsborough, M.A., Enger, C., Selnes, O.A., 1997. Depression and cognitive decline after coronary artery bypass grafting. Lancet 349, 1282-1284.

Musselman, D.L., Evans, D.L., Nemeroff, C.B., 1998. The relationship of depression to cardiovascular disease. Arch. Gen. Psychiatry 55, 580-592.

Nashef, S.A.M., Roques, F., Michel, P., Gauducheau, E., Lemeshow, S., Salamon, R., The EuroSCORE study group, 1999. European system for cardiac operative risk evaluation (EuroSCORE). Eur. J. Cardiothorac. Surg. 16, 9-13.

Peñacoba-Puente, C., Fernández-de-Las-Peñas, C., González-Gutierrez, J.L., Miangolarra-Page, J.C., Pareja, J.A., 2008. Interaction between anxiety, depression, quality of life and clinical parameters in chronic tension-type headache. Eur. J Pain 12, 886-894.

Pirraglia, P.A., Peterson, J.C., Williams-Russo, P., Gorkin, L., Charlson, M.E., 1999. Depressive symptomatology in coronary artery bypass graft surgery patients. Int J. Geriatr. Psychiatry 14, 668-680.

Rubin, D.B., 1987. Multiple Imputation for Nonresponse in Surveys. Wiley \& Sons, New York. 
Scott, K.M., McLaughlin, K., Smith, D.A.R., Ellis, P.M., 2012. Childhood maltreatment and DSM-IV adult mental disorders: comparison of prospective and retrospective findings. Br. J. Psychiatry 200, 469-475.

Solomon, M.B., Herman, J.P., 2009. Sex differences in psychopathology: of gonads, adrenals and mental illness. Physiol. Behav. 97, 250-258.

Spielberger, C.D., Gorsuch, R.L., Lushene, P.R., Vagg, P.R., Jacobs, G.A., 1983. Manual for the State-Trait Anxiety Inventory (Form Y). Manual for the statetrait Anxiety Inventory STAI.

Spindler, H., 2005. Posttraumatic stress disorder in the wake of heart disease: prevalence, risk factors, and future research directions. Psychosom. Med. 67, 715-723.

Thombs, B.D., Bernstein, D.P., Lobbestael, J., Arntz, A., 2009. A validation study of the Dutch childhood trauma questionnaire-short form: factor structure, reliability, and known-groups validity. Child Abus. Negl. 33, 518-523.

Tully, P.J., 2012. Psychological depression and cardiac surgery: a comprehensive review. J. Extra Corpor. Technol. 44, 224-232.

Van der Ploeg, H.M., 1980. Validity of the zelf-beoordelings-vragenlijst (a dutch version of the spielberger state-trait anxiety inventory). Ned. Tijdschr. Psychol Grensgebieden 35, 243-249.

Van Zelst, W., de Beurs, E., Beekman, A., Deeg, D.J.H., Bramsen, I., van Dyck, R., $2003 \mathrm{~b}$. Criterion validity of the self-rating inventory for posttraumatic stress disorder (SRIP) in the community of older adults. J. Affect Disord. 76, 229-235. Van Zelst, W.H., de Beurs, E., Beekman, A.T.F., Deeg, D.J.H., van Dyck, R., 2003a. Prevalence and risk factors of posttraumatic stress disorder in older adults. Psychother. Psychosom. 72, 333-342.

Van Zuiden, M., Geuze, E., Willemen, H.L.D.M., Vermetten, E., Maas, M., Heijnen, C.J., Kavelaars, A., 2011. Pre-existing high glucocorticoid receptor number predicting development of posttraumatic stress symptoms after military deployment. Am. J. Psychiatry 168, 89-96.

Van Zuiden, M., Geuze, E., Willemen, H.L.D.M., Vermetten, E., Maas, M., Amarouchi, K., Kavelaars, A., Heijnen, C.J., 2012. Glucocorticoid receptor pathway components predict posttraumatic stress disorder symptom development: a prospective study. Biol. Psychiatry 71, 309-316.

Vinkers, C.H., Joëls, M., Milaneschi, Y., Kahn, R.S., Penninx, B.W.J.H., Boks, M.P.M. 2014. Stress exposure across the life span cumulatively increases depression risk and is moderated by neuroticism. Depress. Anxiety 9, 1-9,

Viswanath, B., Maroky, A.S., Math, S.B., John, J.P., Cherian, A.V., Girimaji, S.C., Benegal, V., Hamza, A., Chaturvedi, S.K., 2011. Gender differences in the psychological impact of tsunami. Int J. Soc. Psychiatry 59, 130-136.

Wolfe, J.W., Kimerling, R., Brown, P.J., Chrestman, K.R., Levin, K., 1996. Psychometric review of the life stressor checklist-revised. In: Stamm, B.H. (Ed.), Measurement of Stress, Trauma, and Adaptation. Sidran Press, Lutherville, MD. 